\title{
Calcul d'une formule barymétrique adaptée au zébu gobra
}

\author{
par M. CHOLLOU, J. P. DENIS, D. GAUCHET
}

\begin{abstract}
RÉSUMẼ
Les auteurs donnent à partir de l'observation de 7596 couples de poids périmètres-thoraciques relevés chez des zébus Gobra du Sénégal, un tableau de conversion du périmètre thoracique en poids, utilisable directement dans les conditions de travail sur le terrain (enquêtes, suivis d'évolution pondérale...).
\end{abstract}

\section{INTRODUCTION}

Au Sénégal, il est encore impossible d'équiper tout le pays du nombre suffisant de bascules pèse-bétail nécessaires pour un suivi correct des opérations zootechniques. Seules les stations de recherches disposent actuellement d'un matériel utilisable.

Il a donc paru intéressant d'adopter une formule barymétrique pour l'appréciation du poids des animaux, l'opérateur n'ayant besoin pour obtenir le résultat pondéral que d'un mètre ruban et d'une table de conversion. La vulgarisation du procédé, étant donné sa simplicité d'utilisation, pourra ainsi être facilement faite.

\section{MATÉRIEL ET MÉTHODES}

\section{Matériel animal d'étude}

Les données concernent des zébus Gobra mâles et femelles nés au Centre de Recherches Zootechniques de Dahra (Sénégal) de 1965 à 1971. Ces mesures (poids et périmètre thoracique) ont été effectuées tous les 3 mois de la naissance à 5 ans, soit 7596 couples poidspérimètre thoracique.

\section{Choix des mesures}

En fait, les animaux étudiés sont soumis à un grand nombre de mensurations: périmètre thoracique, longueur scapulo-ischiale, hauteur au garrot, hauteur aux sangles, longueur et largeur de la tête, longueur de la croupe et largeur aux hanches. Dans un premier temps, l'examen de la littérature nous a conduits à ne retenir que le périmètre thoracique qui constitue la mesure donnant le maximum de précision. Un autre souci important a été l'utilisation de la méthode la plus rapide et la plus simple possible.

\section{Formulation}

Toujours en vue d'un emploi le plus simple possible, on n'a tenu compte ni de l'âge ni du sexe. L'influence du sexe est, en effet, difficilement séparable d'autres incidences, en particulier nutritionnelles. Or, les animaux Gobra, quelque soit le sexe, sont entretenus dans des conditions tout à fait comparables durant la période de recueil des données. Quant à l'âge, son appréciation en brousse n'est pas toujours très simple à obtenir et, d'autre part, les régimes alimentaires appliqués ne subissent pratiquement pas de variations dans le temps. 
Une étude ultérieure permettra, cependant, liaison entre poids et périmètre thoracique n'est de préciser l'influence de ces différents facteurs: pas linéaire mais de type parabolique (voir âge, sexe, état musculaire et état de gestation graphique).

chez les femelles.

Pour chaque périmètre thoracique le poids moyen et son écart type ont été calculés. La

L'ajustement est effectué à l'aide d'un polynôme du second degré :

$$
\grave{y}=a X^{2}+b X+c .
$$

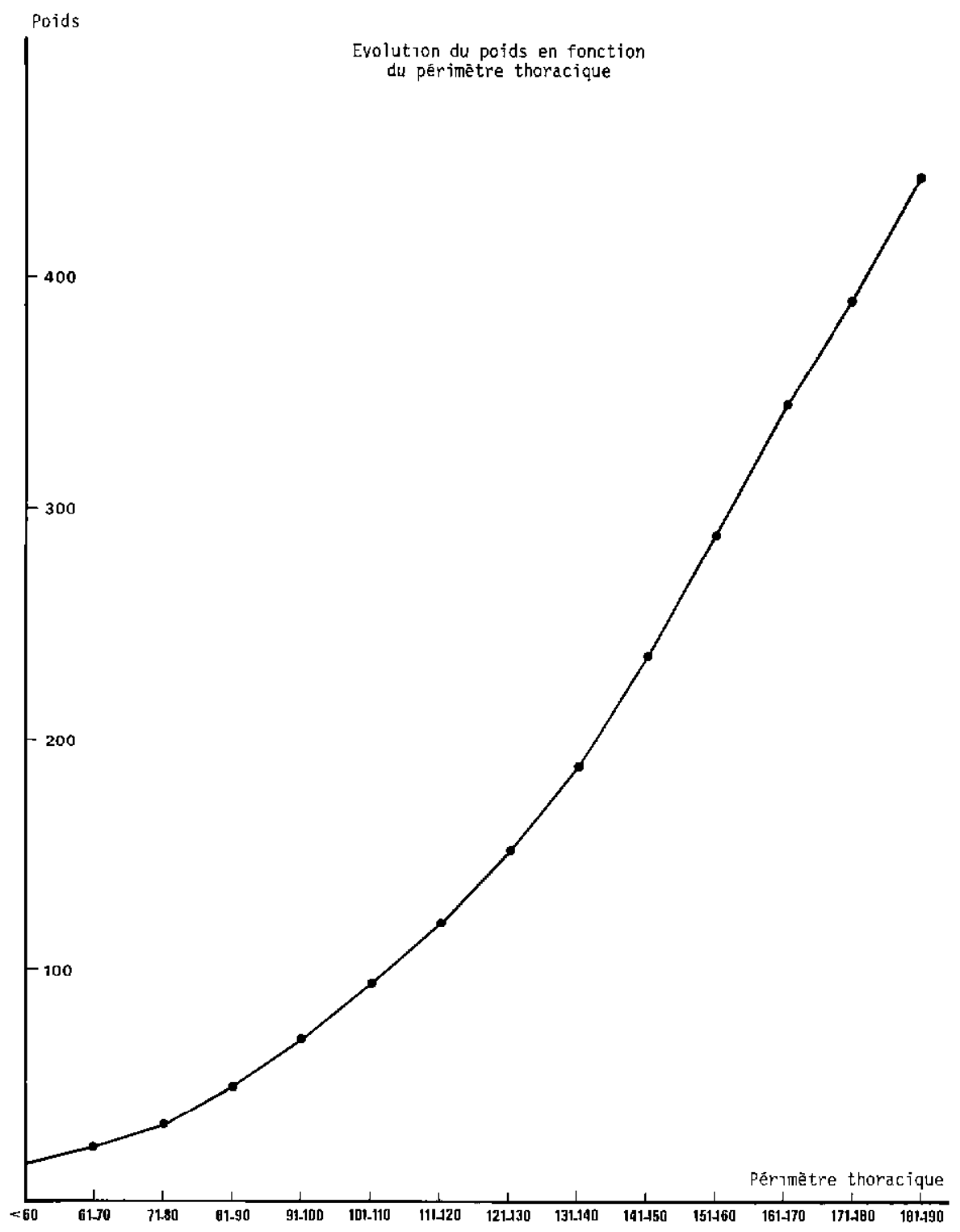

\section{RÉSULTATS}

1. Corrélation entre poids et périmètre thoracique

Le coefficient de corrélation calculé sur 7596 couples poids-périmètre thoracique est de 0,971 avec des limites de confiance à 95 p. 100 de 0,969 et 0,972 .
Ce résultat est très voisin de ceux obtenus chez les taurins européens, toujours supérieurs à 0,9 .

\section{Paramètres de la parabole de régression}

Les paramètres $a, b$ et $c$, estimés par la méthode des moindres carrés, ont les valeurs suivantes: 


$$
\begin{aligned}
& \mathrm{a}=\quad 0,0247 \\
& \mathrm{~b}=-2,5433 \\
& \mathrm{c}=83,9171
\end{aligned}
$$

d'où l'équation :

$$
y=0,0247 x^{2}-2,5433 x+83,3171
$$

où $\mathrm{y}$ représente le poids exprimé en $\mathrm{kg}$ et $\mathrm{x}$ le périmètre thoracique exprimé en $\mathrm{cm}$.

A partir de cette formule, la valeur correspondante du poids a été calculée pour chaque valeur du périmètre thoracique. Cette correspondance poids-périmètre thoracique constitue la table de conversion utilisable sur le terrain (en annexe).

L'ajustement effectué est excellent puisque le $\chi^{2}$ calculé $(10,35)$ est largement compris dans la zone de vraisemblance $(\alpha=0,05$; $\mathrm{N}=136$ ).

Pour estimer la précision de cette conversion pour chaque périmètre thoracique on a tenu compte :

de l'effectif, de l'écart à la moyenne estimée ( $\left.e_{m}\right)$ exprimé en p. 100.

- de l'écart moyen individuel $\left(\mathrm{e}_{1}\right)$ exprimé en p. 100 , et pour l'ensemble des données on obtient :

$$
\begin{gathered}
\mathrm{e}_{\mathrm{m}}=1,69 \text { p. } 100, \\
\mathrm{e}_{\mathrm{i}}=7,46 \text { p. } 100 .
\end{gathered}
$$

L'utilisation d'une bascule pèse-bétail mobile ou fixe en brousse conduit souvent à des erreurs sur les poids aussi importantes, sinon plus, que celles enregistrées par cette méthode barymétrique, du fait de l'indocilité des animaux, des difficultés d'entretien des bascules, etc.

\section{CONCLUSION}

La table de conversion ainsi établie permet

\begin{tabular}{|c|c|c|c|c|c|c|c|c|c|c|c|}
\hline $\begin{array}{l}\text { P.T. } \\
(\mathrm{cm})\end{array}$ & $\begin{array}{c}\mathrm{P} . \\
(\mathrm{kg})\end{array}$ & $\begin{array}{l}\text { P.T. } \\
\text { (cm) }\end{array}$ & $\begin{array}{c}\mathrm{P} \\
(\mathrm{kg})\end{array}$ & $\begin{array}{l}\text { P.T. } \\
\text { (cm) }\end{array}$ & $\begin{array}{c}P . \\
(\mathrm{kg})\end{array}$ & $\begin{array}{l}\text { P.T, } \\
\text { (cin) }\end{array}$ & $\underset{(\mathrm{kg})}{\mathrm{P}}$ & $\begin{array}{l}\text { P.T. } \\
(\mathrm{cm})\end{array}$ & $\begin{array}{c}\text { P. } \\
(\mathrm{kg})\end{array}$ & $\begin{array}{l}\text { P.T. } \\
\text { (CDO) }\end{array}$ & $\underset{(\mathrm{kg})}{\mathrm{P}}$ \\
\hline 54 & 18,5 & 77 & 35 & 100 & 77 & 123 & 145 & 146 & 239 & 169 & 360 \\
\hline 55 & 19 & 78 & 36 & 101 & 79 & 124 & 148 & 147 & 244 & 170 & 365 \\
\hline 56 & 19 & 79 & 37 & 102 & 81 & 125 & 152 & 148 & 249 & 171 & 371 \\
\hline 57 & 19 & 80 & 39 & 103 & 84 & 126 & 156 & 149 & 253 & 172 & 377 \\
\hline 58 & 19,5 & 81 & 40 & 104 & 87 & 127 & 159 & 150 & 258 & 173 & 383 \\
\hline 59 & 20 & 82 & 41 & 105 & 89 & 128 & 163 & 151 & 263 & 174 & 389 \\
\hline 60 & 20 & 83 & 43 & $106 ́$ & 92 & 129 & 167 & 152 & 268 & 175 & 395 \\
\hline 61 & 20,5 & 84 & 45 & 107 & 95 & 130 & 171 & 153 & 273 & 176 & 401 \\
\hline 62 & 21 & 85 & 46 & 108 & 97 & 131 & 175 & 154 & 278 & 177 & 408 \\
\hline 63 & 21,5 & 86 & $4 B$ & 109 & 100 & 132 & 179 & 155 & 283 & 178 & 414 \\
\hline 64 & 22,5 & 87 & 50 & 110 & 103 & 133 & 183 & 156 & 288 & 179 & 420 \\
\hline 65 & 23 & 88 & 51 & 111 & 106 & 134 & 187 & 157 & 293 & 180 & 426 \\
\hline 66 & 23,5 & 89 & 53 & 112 & 109 & 135 & 191 & 158 & 299 & 181 & 433 \\
\hline 67 & 24,5 & 90 & 55 & 113 & 112 & 136 & 195 & 159 & 304 & 182 & 439 \\
\hline 68 & 25 & 91 & 57 & 114 & 115 & 137 & 199 & 160 & 309 & 183 & 446 \\
\hline 69 & 26 & 92 & 59 & 115 & 118 & 138 & 203 & 161 & 315 & 184 & 452 \\
\hline 70 & 27 & 93 & 61 & 116 & 121 & وق 1 & 208 & 162 & 320 & 185 & 459 \\
\hline 71 & 28 & 94 & 63 & 117 & 124 & 140 & 212 & 163 & 326 & 186 & 465 \\
\hline 72 & 29 & 95 & 65 & 118 & 128 & 141 & 216 & 164 & 331 & 187 & 472 \\
\hline 73 & 30 & 96 & 67 & 119 & 131 & 142 & 221 & 165 & 337 & 188 & 479 \\
\hline 34 & 31 & 97 & 70 & 120 & 134 & 143 & 225 & 166 & 342 & 189 & 486 \\
\hline 75 & 32 & 98 & 72 & 121 & 138 & 144 & 230 & 167 & 348 & 190 & 492 \\
\hline 76 & 33 & 99 & 74 & 122 & 141 & 145 & 234 & 168 & 354 & & \\
\hline
\end{tabular}
d'assurer avec une précision tout à fait suffisante un suivi pondéral plus rapide et plus fréquent sur un nombre d'animaux plus élevé que par un système classique de pesées.

Tableau de conversion entre le périmètre thoracique (P.T.)-1es poids (P.). 


\section{SUMMARY}

Calculation of a barymetrical formula adapted to Gobra zebu cattle

Starting from the observation of 7596 ratios of weight/thoracic perimeter recorded in zebu cattle in Senegal, the authors have drawn conversion tables of thoracic perimeter into weight, utilizable directly in field working conditions (surveys, followups of weight evolution).

\section{RESUMEN}

\section{Cálculo de una fórmula barimetrica adaptada al cebú Gobra}

A partir de la observación de 7596 pares de pesos-perímetros torácicos recogidos en cebues Gobra del Senegal, los autores dan una tabla de conversión del perímetro torácico en peso, utilizable directamente en las condiciones de trabajo sobre terreno (encuestas, observaciones seguidas de evolución ponderal...). 